\title{
An Intelligent Remote Control System Based on a Wireless Sensor Network
}

\author{
http://dx.doi.org/10.3991/ijoe.v12i02.5048 \\ WEI Kaibin \\ Northwest A\&F University, Shaanxi, China
}

\begin{abstract}
A remote control system is mainly used for industrial applications such as the wireless control of factory equipment and to monitor factory environment parameters, like temperature, humidity, light intensity. This paper explores the current development of a wireless sensor network (WSN), constructs an overall architecture of a remote control system on the basis of an RS485 platform, and analyzes and designs an intelligent remote control system. Finally by testing the system, it was proven to meet the specific needs of factory environment parameters monitoring. The system can achieve all kinds of physical parameter monitoring requirements, such as humidity and light density, from a remote device. This remote control system based on a wireless sensor network provides a safety monitoring environment for operators.
\end{abstract}

Index Terms-Intelligent system, Remote Control, Wireless Sensor Network

\section{INTRODUCTION}

\section{A. The Development of the Wireless Sensor Network}

With the rapid development of wireless communication, sensors, integrated circuits, and micro-electronic mechanical system (MEMS) technology and with the increasing maturity of the technologies as well as the low cost and low power consumption, the multi-functionality of the mass production of micro sensors is possible [1-3]. These sensors, usually in small volume, integrate information collection and have wireless communication and data processing and other functions. A wireless sensor network is composed of tens to thousands of micro sensor selforganizing networks with a dynamic topology structure. Its purpose is to collaborate, collect and process the monitoring object information of the network coverage area, and then send it to the observer. Because the micro sensor is small in volume and light weight, some of them like dust floating in the air, people call wireless sensor networks (WSN) "Smart Dust." The network spreads around to sense real-time changes in the physical world and changes its state and function according to the operator's command.

A wireless sensor network (WSN) is a kind of non- center node full distribution system. Because of the large number of the sensor nodes, the sensors can only be dispatched in a random way, and the sensors are densely deployed in the monitoring area [4]. These sensor nodes are integrated with the sensors, the data processing unit, and the communication module through the wireless, selforganizing network system [5]. With all kinds of built-in sensors in the sensor nodes, they can measure the parame- ters of heat, infrared, sonar, radar, and seismic signals. The sensor nodes can detect information including temperature, humidity, noise, light intensity, pressure, composition of soil, size of a moving object, speed and direction, and many other parameters interesting in physics. Good coordination between the sensor nodes is very important. Usually the global tasks are done by local data exchange. Due to the energy-saving requirements of sensor networks, a multi-jump, peer-to-peer communication mode rather than a traditional single jump, master-slave communication mode is more suitable for a WSN. At the same time the peer-to-peer communication mode can effectively avoid signal fading and interference and all sorts of problems in the process of long distance wireless signal transmission. Through the gateway, the sensor network also can be connected to the existing network infrastructure (such as the Internet, mobile communication network); thus, the information collected can be used by remote terminal users. Because a WSN involves sensor technology, network communication technology, wireless transmission technology, embedded technology, distributed information processing technology, microelectronics manufacturing technology, software technology, it is a highly cross disciplinary and an emerging and frontier research field.

\section{The Overall Architecture Of The Remote CONTROL SYSTEM}

The whole system mainly consists of two parts: one is the PC simulator (Test Program), and the other is the Wireless Unit (Wireless Unit). The basic structure is shown in Figure 1.

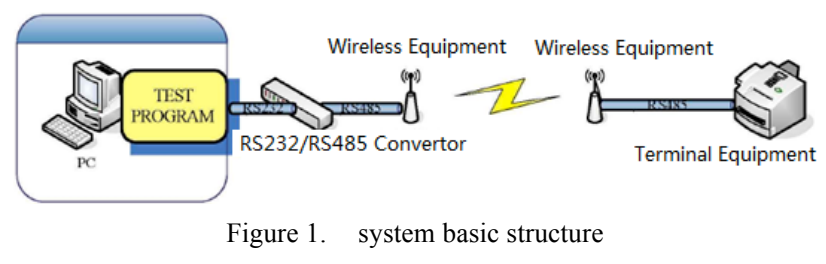

Because the out port of the PC is an RS232, the system uses the cable connection mode RS485 as a standard, so the cable connection between the simulator and wireless unit must go through the RS232 / RS485 converter. The rest are used to connect other cable electrical standards for the RS485 bus standard. The connection between the wireless unit connected through the wireless communication mode is IEEE802.15.4 standard $2.4 \mathrm{GHz}$ frequencies. The frequencies used in the field of scientific research, medical treatment, do not need to apply to the administrative departments of radio for radio frequency. Simulator is an application software based on the Windows operating 
system that provides support for the RS232 and RS485 bus, with a friendly graphical user interface. It is used to simulate the network based on the RS485 bus interface device (Network Interface Unit). It is developed with $\mathrm{C}++$ or C\# language. Through the Test Program simulation of the network interface unit, it can simplify the development process. The current development has focused on a wireless unit. The wireless unit's main function is to receive the RS485 bus transmission data and then send it out with a CC2420 frequency unit. At the same time, the wireless unit will receive data from other wireless units and then the data received through the RS485 bus simulator.

\section{A. Remote system overall construction and software design}

The wireless unit uses an embedded system as the control unit. The wireless unit shall have the following basic functions:

(1) Provide the RS485 bus interface to connect to the emulator.

(2) Provide wireless radio frequency units to communicate with other wireless units.

(3) Use a microprocessor unit for receiving data from the RS485 bus simulator, so the unit can forward data through the wireless unit.

The basic process of the wireless unit is shown in Figure 2 .

\section{B. Cable network structure and design}

The cable network is still necessary in the connection from a PC to the wireless unit and the network terminal, the cable network uses an RS485 bus.

The basic characteristics of RS485 communication

RS485 adopts differential signal negative logic: $+2 \sim$ $+6 \mathrm{~V}$ stands for $0,-6 \sim-2 \mathrm{~V}$ stands for 1 . The RS485 has a two or four wire system for wiring. A four wire system can only realize point-to-point communication, which is rarely used now. In this project, we used a 2 wire connection mode. This kind of connection mode is the bus topology structure, so on the same bus, the mode can be articulated in 32 nodes. The RS485 communication network generally uses a master-slave communication mode, that is, a host with multiple slave machines. In many cases, the connection to the RS485 communication link is simply a pair connected to each interface of "A" and "B." It ignores the signal ground connection. This kind of connection method works on many occasions, but it has a lot of hidden dangers. There are two reasons:

(1) The common-mode interference problem:

An RS485 interface uses differential mode transmission signals. It does not need a relative reference point to detect signals; the system can detect the potential difference between the two lines. But people often ignore that the transceiver has a certain common mode voltage range. The RS485 transceiver of common-mode voltage range is $-7 \sim+12$ V. Only with the above conditions can the entire network work normally. When the common-mode voltage is beyond this range, it can affect communication stability and reliability, and even damage the interface.

(2) The EMI problem:

Sending drivers sending the output signal of a common mode requires a return path. Without a low resistance return pathway (signal), the signals will return in the form

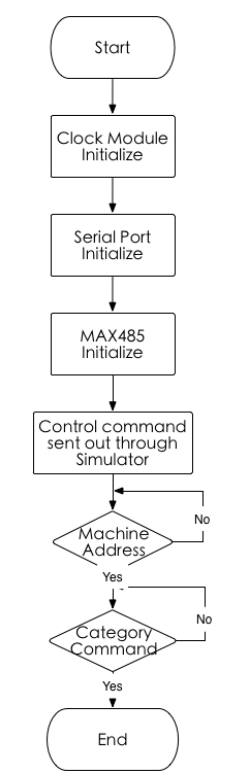

Figure 2. The flow chart of the wireless unit

of radiation to the source side. The whole bus would be like a giant antenna to radiate electromagnetic waves. Due to PCs having an RS232 interface by default, there are two ways to get the PC computer RS485 circuit:

a. Through the RS232 / RS485 converter circuit, the PC serial port RS232 signal converts into RS485 signals. For complicated industrial environments, the converter should choose surge protection products with isolating functions.

b. Through a PCI multi-serial port card, the signals will directly output to the RS485 expansion card. In general situations, the cable uses an ordinary twisted pair. In higher demand environments, the cable can use a shield coaxial cable. With the RS485 interface, for a specific transmission line from the RS485 interface to the load, the maximum allowed cable length is inversely proportional to the signal transmission baud rate. The cable length is mainly affected by distortion of the signal and noise. Theoretically, RS4885's maximum transmission distance can reach $1200 \mathrm{~m}$, but in practice, the transmission distance is shorter than $1200 \mathrm{~m}$. The transmission distance depends on the surrounding environment. In the process of transmission, adding photoelectric converters as relays to both ends of the transmission to increase the transmission distance is put into the dawn. The multimode fiber transmission distance is $5 \sim 10 \mathrm{~km}$, and a single mode fiber can reach $50 \mathrm{~km}$.

2. RS-485 interface standard

Transmission: Differential

Transmission media: twisted pair

Standard Section Points: 32

Farthest transmission distance: $1200 \mathrm{~m}$; common-mode voltage maximum and minimum value: $12 \mathrm{~V} ;-7 \mathrm{~V}$

Differential Input Range: $-7 \mathrm{~V} \sim 12 \mathrm{~V}$

The receiver input sensitivity: $\pm 200 \mathrm{mV}$

Receiver input impedance: $\geqslant 12 \mathrm{~km}$

3. RS-485 connection diagram circuit

A MAX485 interface chip is a Maxim RS-485 chip. Working with a single $5 \mathrm{~V}$ power supply, the rated current of $300 \mu$ A using half-duplex communication, it completed the conversion of a TTL level to an RS485 level function. The MAX485 chip and pin are very simple, internal- 
ly containing a driver and receiver. RO and DI, respectively, are the output of the receiver and input of the drives. When connected to the micro controller, it only needs to connect to the MCU's RXD and TXD; the /RE and DE ends are for receiving and sending. When /RE is at logic 0 , the device is in the receiving state, and when the $\mathrm{DE}$ is at logic 1 , the device is in the sending state because the MAX485 works in a half-duplex state, so just one pin of the MCU can control the two pins. A and B are the differential signals for the receiving and sending end, when the pin level of $\mathrm{A}$ is higher than $\mathrm{B}$, the sending data is 1 . When the A level is lower than $\mathrm{B}$, the sending data is zero. Wiring is very simple when connected to the microcontroller. Only a signal control MAX485 receiving and sending is needed. At the same time, adding a matching resistance between the A and B sides, the resistance generally is optional at $100 \Omega$. The specific circuit connection is shown in Figure 3.

\section{Stesting Of The Remote System}

\section{A. System Network with RS485 bus}

Network topology is generally used for termination to the total linear structure, which does not support ring or star networks. To build the network, we should pay attention to the following points:

(1) Connect each node with a twisted pair cable. From the bus to each node of the lead, the wire length should be as short as possible to make the lead wire's influence on the reflection signals on the bus as small as possible. Some network connections, even though they are not correct but are short distance low rate cases, could still work. As an extension of the communication distance or an increase of the communication rate, the impact will be more and more serious. The main reason is that the signal at the end of each branch overlaps the original signal after reflection and will cause a drop in the quality of the signal.

(2) The bus should be paid attention to for the continuity of the characteristic impedance and the impedance discontinuity that occurs in signal reflection. The following ways will produce the discontinuity: different stages of the bus take a different cable, a bus install has many tightly connected receivers, or there is a long branch line drawn to the bus.

(3) In the process of constructing an RS485 network, another thing need to pay attention to is the problem of terminal load resistance. In the case of a small amount of equipment or a short distance, without adding the terminal load resistance, the network can work well. But with an increase of distance, the performance of the system will be reduced. In theory, sampling at the end of each receives a data signal. As long as the reflected signal attenuation at the beginning of the sample is low enough, the two end will match. But it is difficult to sample in a practical application. The general matching method of terminal resistance uses the terminal resistance method; RS485 should be in the beginning and end of the bus cable and terminal resistance. The termination resistor in the RS485 network is $120 \Omega$, which equals the resistance of the characteristic impedance of the cable because most of the characteristic impedance of the twisted-pair cable is $100 \Omega \sim 120 \Omega$. This matching method is simple and effective, but there is one drawback. The matching approach will consume more power, so a system with strict power limitations is not suitable for this method. Another match-

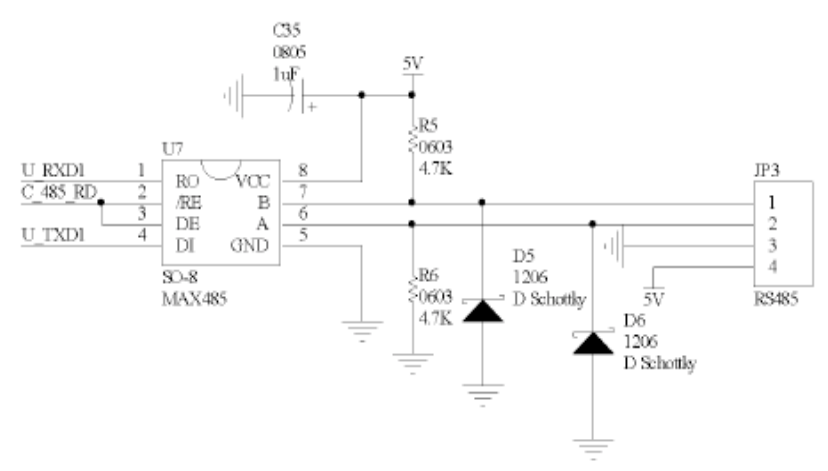

Figure 3. RS485 circuit connection

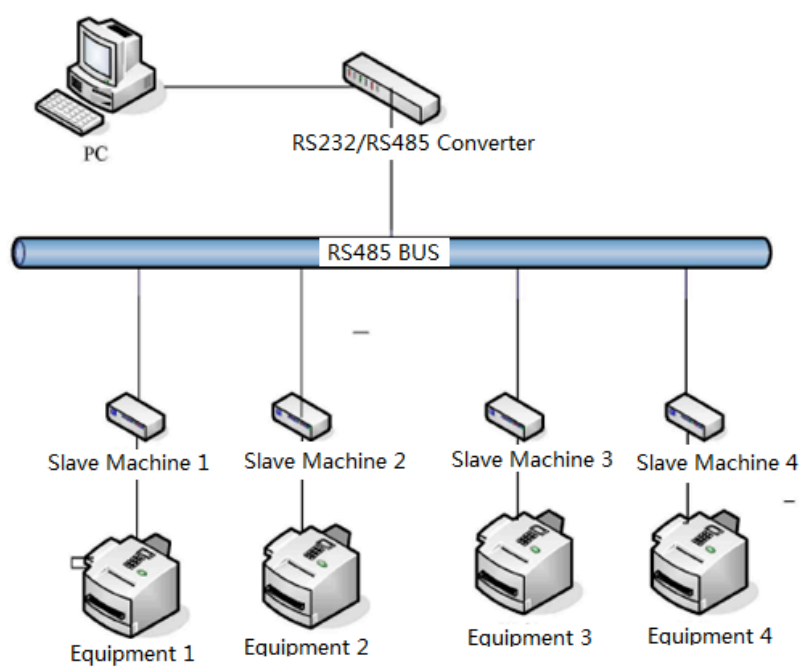

Figure 4. The structure of wireless Equipment connect to the network

ing way to compare saved electricity is RC matching. Using a capacitance $C$ partition can greatly save the DC component of the power. But the capacitance value of $\mathrm{C}$ is difficult to determine; it needs to compromise between power consumption and matching quality. Still there is a kind of diode matching method. This solution is not to achieve a real "match," but with the clamp diode, it can weaken the reflected signal quickly to improve the signal quality. This method has a remarkable energy saving effect. RS485 is a popular way to construct a network. It is characterized by simple implementation. And now there are a large number of instruments supporting RS485s. Especially in the oil industry, RS485/MODBUS is unifying the whole industry, as contractors are in favor of RS485/MODBUS, which is a very simple, original HART instrument Buying a switch port is difficult, and the instrument is very expensive. Transformation of an RS485 interface is much cheaper and has a great variety of instrument supports. At least in the low-end market, RS485/MODBUS is the main way to construct a network and the structure will not change.

The structure of wireless equipment connected to the network is shown in Figure 4.

\section{B. System testing}

The local control system works at a low voltage of $3 \sim 5 \mathrm{~V}$. By sending commands to the emulator and transmitting the signal to the wireless unit, signals will be sent to the wireless unit. In the receiving device on the wireless network, the port voltage depends on the received signal level. The test flow chart is shown in Figure 5. 


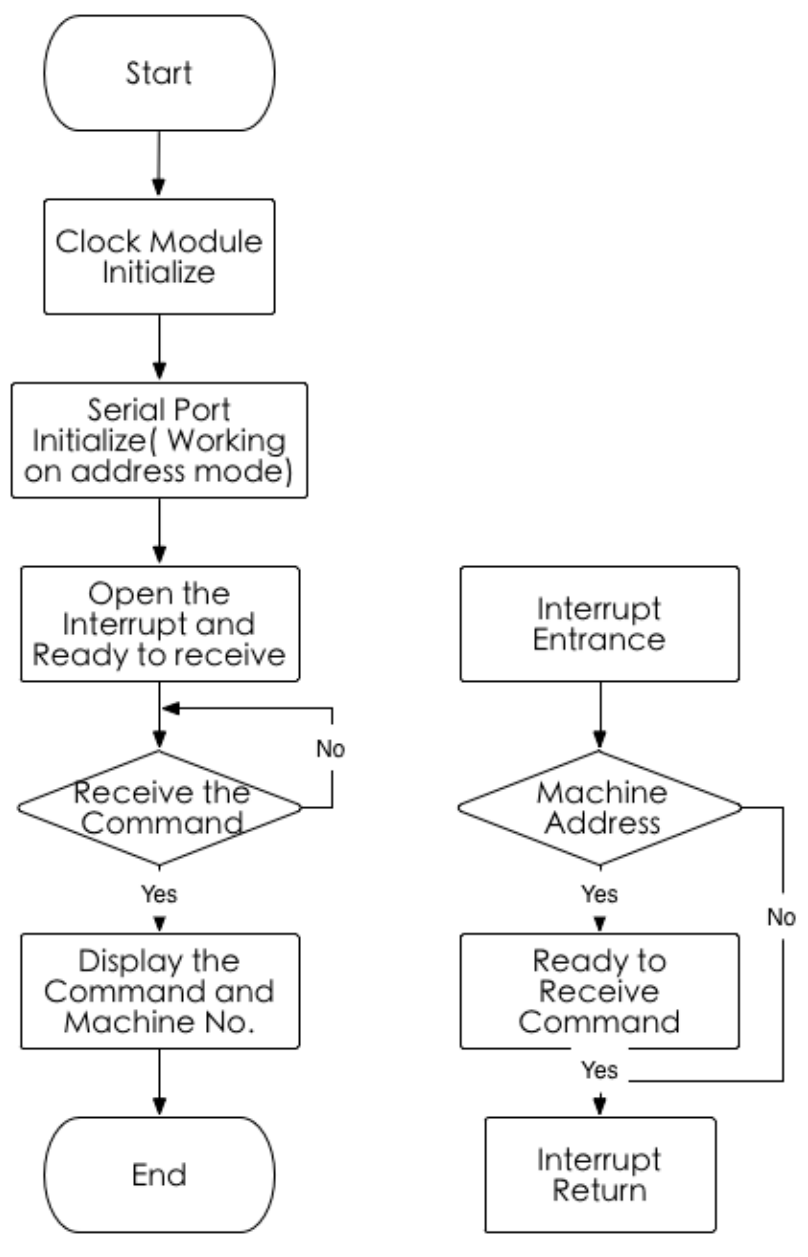

Figure 5. System testing flow chart

\section{CONCLUSION}

This paper studies a remote control system based on a wireless sensor network (WSN) on the basis of practical experience building the basic platform of a remote system that realizes the basic wireless sending and receiving of data to control a remote device. It also describes the cable part of the whole wireless network. This paper realizes a remote network of multiple devices and the simulator's matching function. With the RS485 bus, the network can easily be constructed on the wireless side; on the other side, the simulator can easily be operated through the proximate control command to achieve one-to-many and many-to-many control of the remote device.

\section{REFERENCES}

[1] Kong, L., \& Wang, H. (2013). Wireless sensor networks based on intelligent street lighting system. Electronic Science \& Technology.

[2] Lv Xiaoying, Chen Shaohua, Shi Gui, and Zhou di (2014). The intelligent monitoring system of urban rail train based on wireless sensor network.

[3] Li Xuemei (2013). Study on coal mine safety monitoring system based on wireless sensor, coal technology, 32 (5), 8385.DOI:10.3969/j.issn.1008-8725.2013.05.040.

[4] Li Chao. (2014). The design and application of sound recognition sensor. Sensor and micro system, 33 (12). DOI:10.13873/J.10009787 (2014) 12-0051-03.

[5] Leccese, F. (2013). Remote-control system of high efficiency and intelligent street lighting using a zigbee network of devices and sensors. Power Delivery IEEE Transactions on, 28(1), 21-28. http://dx.doi.org/10.1109/TPWRD.2012.2212215

\section{AUTHOR}

WEI Kaibin is with Northwest A\&F University, Shaanxi, CO712100, China.

Submitted 17 September 2015. Published as resubmitted by the author 23 January 2016. 